Southern Illinois University Edwardsville SPARK

Spring 5-10-2019

\title{
Heart Failure Knowledge among CNAs in a Skilled Nursing Facility: A Pilot Project
}

Amanda McDannald

Follow this and additional works at: https://spark.siue.edu/dnpprojects

Part of the Adult and Continuing Education Commons, and the Nursing Commons

\section{Recommended Citation}

McDannald, Amanda, "Heart Failure Knowledge among CNAs in a Skilled Nursing Facility: A Pilot Project" (2019). Doctor of Nursing Practice Projects. 52.

https://spark.siue.edu/dnpprojects/52

This DNP Project is brought to you for free and open access by the School of Nursing at SPARK. It has been accepted for inclusion in Doctor of Nursing Practice Projects by an authorized administrator of SPARK. For more information, please contact magrase@siue.edu. 
Heart Failure Knowledge among CNAs in a Skilled Nursing Facility: A Pilot Project

\section{Introduction of the Problem}

Heart failure (HF) is the leading cause of hospitalization among patients over the age of 65 years old. Yancy et al. (2013) identified heart failure specific education, regular physical activity, low sodium diets, and volume status management as some factors which decrease rehospitalization rates. Many patients with HF are transitioned to skilled nursing facilities (SNF) following hospital discharge. The 30-day readmission rates are higher at SNFs than those discharged home (Jurgens et al., 2015). Improved adherence to guideline-recommended care shows promising advances in an attempt to decrease HF-related hospital readmissions (Yancy et al., 2013).

\section{Literature Review}

Licensed Practical Nurses (LPNs) and Certified Nursing Assistants (CNAs) comprise the majority of SNF staffing. Boxer et al. (2012) conducted a study in four nursing homes over two years that suggested staff members were aware of HF being a common condition among admitted patients but did not know that there were national management guidelines or care recommendations. Staff also reported that they did not receive HF-specific education or incorporate HF-specific interventions into daily patient care.

Controllable barriers to avoid HF-related rehospitalization in SNFs have been reported and include a lack of HF care knowledge and interprofessional communication skill (Heckman et al., 2016). A quality improvement project that addresses barriers such as facility regulatory or process conflicts and continued staff education may help in providing optimal care. Those admitted to an SNF already have complex comorbidities and progressed disease states that require care regimens rooted in evidence-based practice. Staff education and experience 
deficiencies, due to high turnover and limited access to providers, may contribute to suboptimal symptom recognition and early intervention. This is why it is important to create sustainable orientation programs and reoccurring training sessions.

Volume status management is highly effective in providing quality care to those with HF. Increased weight, peripheral edema, and shortness of breath are traditional methods of evaluating volume status effectively (McDonald, Wilkinson, \& Ledwidge, 2015). Certified Nursing Assistants are in constant contact with the patient and are in the position to alert nurses of increased weight, edema, voiding patterns, and activity tolerance (Jurgens et al., 2015). Sodium balance has also been closely associated with volume status and should be restricted in HF patients when they are symptomatic (Jurgens et al., 2015). There is no evidence to suggest that skilled nursing facilities are currently required to offer a low sodium menu option. Supporting residents in choosing a diet low in sodium will help patients adhere to recommendations (Nieuwenhuis, Jaarsma, van Veldhuisen, Postmus, and van der Wal, 2012).

\section{Project Methods}

The purpose of this project was to develop a sustainable HF educational program specifically for SNF staff to provide a stronger knowledge base. This educational intervention focused on improving the CNA's HF care knowledge through training offered at orientation and annual competency testing.

\section{Method}

Assessment of perceived knowledge gaps and available education were reviewed. Based on current evidence and the American College of Cardiology, American Heart Association, and Heart Failure Society of America (ACC/AHA/HFSA) guidelines, computer-based modules were developed. Module 1 offered basic HF knowledge and an introduction to HF necessary care. 
Module 2 focused on specific guideline-recommended care measures such as modifying lifestyle choices and completing daily tasks necessary to manage HF in the SNF environment. Resident lifestyle choices such as diet and daily activity are some modifiable options that the SNF staff may be able to negotiate with proper training. Daily tasks specific to HF patients include obtaining a daily weight correctly and monitoring the use of compression stockings. Both modules emphasized recognizing crucial information that would need to be reported. The modules were then reviewed by HF experts who offered feedback, and revisions were made. A written 10 question pretest and posttest was developed and given prior to and following educational module review. Hands-on competencies were developed which included proper weight assessment, application of compression stockings, volume status monitoring, and low sodium diet recommendations. The educational program was implemented during the annual SNF competency fair.

\section{Setting and Participants}

This facility has 90 beds with a diverse patient population with at least two comorbidities, requiring skilled nursing, and the majority of patients are over the age of 65 years. This facility is affiliated with a larger organization, consisting of 35 facilities throughout the Midwest. Currently, four patients maintain a primary diagnosis, and several carry a secondary diagnosis of HF. There are five full-time registered nurses, 31 full-time license practice nurses, and 48 full-time certified nursing assistants. In this pilot institution, only CNAs were invited to participate. Staff were directed to attend a mandatory skills day through the facility protocol. Participants were recruited while in attendance of that event. No prior notification for the project was necessary. The Southern Illinois University Edwardsville Institutional Review Board approved this project proposal through the expedited procedure. 


\section{Evaluation}

HF quality measures included in this project were daily weight, volume status, compression stocking utilization, level of activity tolerance, and recognition of low sodium food choices. Knowledge-based questions were presented to the staff and answers were counted as correct or incorrect. Pretests and Posttest were graded manually and checked by another team member for mistakes. Answers for each question were entered into SPSS software version 19.1 to determine if the computer-based educational session improved clinical knowledge demonstrated through pretest and posttest answers presented through constructed scenario questions. Each question was analyzed for frequency of correct responses, mean score, and range. The difference between total correct scores on the pretest and posttest was examined using Student $t$ testing. Questions that were more frequently answered incorrectly were identified. Skill competency was also evaluated by a proctor to determine if the measures were applied appropriately based on established criteria. The frequency of participants passing the competency the first time and the competencies that most frequently required remediation were identified.

A total of 34 CNAs participated in the program. SNF experience ranged from 6 months to 17 years working as a CNA. Pretest scores ranged from 4 to $10(M=7.8$, SD 1.4$)$ and posttest scores ranged from 6 to $10(M=8.9$, SD 1.0) demonstrating a significant improvement in knowledge $(p=.000)$. Participants struggled with understanding symptoms of fluid volume overload and recognition of subtle symptoms of patient decline. Participants also struggled with medical terminology, including terms such as dyspnea. Hands-on competencies revealed some participants had difficulty with identifying steps for proper weight measurement and low sodium food choices.

Resources appreciated throughout this project were the educational materials and 
participants that were readily available during the implementation phase. Education materials utilized were desks, chairs, computers, printers, pens, and paper. The pilot SNF also had access to the larger organizations educational tools such as mannequins for the compression stocking station. Candidates for participation were present at the facility since the implementation coincided with their annual skills day. Risk/threats noted included the time constraint and areas identified for improvement throughout the implementation phase. For this pilot study, it was beneficial to implement the project on the same day as the skills day. However, with this large amount of material to cover within the intervention, it would be beneficial for participants to complete the computer-based educational session one to seven days prior to the skill-based competency demonstration. Additional proctors during the implementation would also allow for different stations to accommodate each HF measure and may lead to better time management and workflow. During the pilot, only CNAs were invited to participate. The inclusion of all nursing staff would strengthen continuity of care through collaboration and reinforce the aim to better serve patients with HF based on ACC/AHA/HFSA guidelines.

\section{Impact on Practice}

The immediate impact of this educational intervention at the pilot institution positively demonstrated improved test scores based on HF-specific knowledge and skill. The Director of Nursing (DON) of the facility reported positive feedback from participants noting they appreciated the training and were encouraged to begin utilizing their new skills. With continued implementation, the improved HF knowledge and skill will potentially facilitate earlier detection of changes in symptoms and improved management of this patient population. All materials utilized throughout the intervention are attached in the appendices making this project easily replicated. The initial aim of this intervention was to ensure sustainability. This project was 
created with the intention of being easily adaptable to other SNFs and further the reach of the positive impact experienced at the pilot institution.

\section{Conclusions}

HF education is important for all caregivers across the nursing continuum. As HF care continues to transition to SNFs, timely staff education is critically important to ensure optimal patient care and outcomes. The initial impact of the pilot study demonstrated positive outcomes and improved knowledge and skill of the nursing staff. Further study is warranted that explores the implementation of this educational program in other SNFs and assessment of long-range outcomes.

\section{Author Contact Information}

Amanda McDannald, RN, BSN, CCRN

Email: amdann@siue.edu 